\title{
Effects of Long-Term Culture on Human Embryonic Stem Cell Aging
}

\author{
Xiaoyan Xie, ${ }^{1-4}$ Asimina Hiona, ${ }^{1,2}$ Andrew Stephen Lee, ${ }^{1,2}$ Feng Cao, ${ }^{1,2}$ Mei Huang, ${ }^{1}$ Zongjin Li, ${ }^{1,2}$ \\ Athena Cherry, ${ }^{5}$ Xuetao Pei, ${ }^{4}$ and Joseph C. Wu ${ }^{1-3}$
}

In recent years, human embryonic stem (hES) cells have become a promising cell source for regenerative medicine. Although hES cells have the ability for unlimited self-renewal, potential adverse effects of long-term cell culture upon hES cells must be investigated before therapeutic applications of hES cells can be realized. Here we investigated changes in molecular profiles associated with young ( $<60$ passages) and old ( $>120$ passages) cells of the H9 hES cell line as well as young ( $<85$ passages) and old ( $>120$ passages) cells of the PKU1 hES cell line. Our results show that morphology, stem cell markers, and telomerase activity do not differ significantly between young and old passage cells. Cells from both age groups were also shown to differentiate into derivatives of all 3 germ layers upon spontaneous differentiation in vitro. Interestingly, mitochondrial dysfunction was found to occur with prolonged culture. Old passage cells of both the H9 and PKU1 lines were characterized by higher mitochondrial membrane potential, larger mitochondrial morphology, and higher reactive oxygen species content than their younger counterparts. Teratomas derived from higher passage cells were also found to have an uneven preference for differentiation compared with tumors derived from younger cells. These findings suggest that prolonged culture of hES cells may negatively impact mitochondrial function and possibly affect long-term pluripotency.

\section{Introduction}

$\mathrm{H}$ UMAN EMBRYONIC STEM (hES) cells can differentiate into every somatic cell type of the human body and possess the capacity for unlimited replication [1]. As a result, beginning with their isolation in 1998 by Dr. James Thomson, these cells have been considered a leading candidate for a donor cell source in cell replacement therapy. Numerous articles have since demonstrated the potential therapeutic use of hESderived cells in the treatment of diseases affecting the heart $[2,3]$, brain [4,5], pancreas [6], liver [7], and bone marrow [8,9]. Current models of cell replacement therapy used in clinical trials can require billions of cells to achieve optimal effect in patients $[10,11]$. Because available federally approved $\mathrm{hES}$ cell lines are limited, it is likely that repeated and prolonged passaging of hES cells will be necessary for clinical applications of hES cells to be realized. Therefore, it is critical to determine whether long-term in vitro cell culture can adversely affect their capacity to participate effectively in cell regeneration therapy.

Senescence is a process that affects all somatic cells of human body and has traditionally been characterized by telomere shortening, accumulation of nuclear mutation, epigenetic silencing, and mitochondrial dysfunction, the overall effect of which produces the loss of function $[12,13]$. Recently, adult stem cell senescence has also come under scrutiny [14]. hES cells are generally considered to be resistant to replicative senescence. A number of studies have demonstrated that ES cells not only continue to replicate, but also maintain constant telomere length and undergo lower rates of genomic mutation than their somatic counterparts even after prolonged in vitro replication extending into 1 year or longer [15-17]. Stem cells grown in culture for such periods have also been shown to retain normal karyotypes [17-19] and epigenetic stability [20-22], but several recent articles have disputed this claim [23-25].

In our experience, very late passage hES cells have been observed to have a reduced ability to differentiate into derivatives of all 3 germ layers, which may affect their therapeutic potential. To document this reduction in pluripotency and determine whether these changes are associated with replicative senescence, we investigated the proliferation and differentiation of young and old passage hES cells, and

\footnotetext{
${ }^{1}$ Department of Medicine, ${ }^{2}$ Molecular Imaging Program at Stanford, ${ }^{3}$ Institute for Stem Cell Biology and Regenerative Medicine, ${ }^{5}$ Department of Pathology, Stanford University School of Medicine, Stanford, California.

${ }^{4}$ Stem Cells and Regenerative Medicine Lab, Beijing Institute of Transfusion Medicine, Beijing, China.
} 
intracellular indices of aging such as mitochondrial function, telomerase activity, and chromosomal stability.

\section{Materials and Methods}

\section{Culture of hES cells}

H9 hES cells (WiCell) and PKU1 hES cells (non-federalapproved hES cells, a gift from Peking University) [26] were cultured on a feeder layer of irradiated mouse embryonic fibroblasts using hES cell culture medium consisting of $80 \%$ Dulbecco's modified Eagle's medium (DMEM)/F-12 (Invitrogen), 20\% knock-out serum replacement (Invitrogen), $1 \mathrm{mM}$ L-glutamine, $1 \%$ nonessential amino acids, $0.1 \mathrm{mM}$ $\beta$-mercaptoethanol, and $8 \mathrm{ng} / \mathrm{mL}$ basic fibroblast growth factor (Invitrogen). Cells were disassociated with Collagenase IV (Invitrogen) every 4-6 days. Before analysis, cells were moved to a Matrigel (hES cell-qualified Matrix; BD Biosciences)-coated plate and cultured for 2 passages with mTeSR feeder-free medium (StemCell Technology). H9 cells having undergone $<60$ passages or $>120$ passages were defined as young or old passage cells, respectively. PKU1 cells having undergone $<85$ passages or $>120$ passage were defined as young or old passage cells, respectively.

\section{Immunofluorescence}

hES cell colonies plated on chamber slides (Lab-Tek, Nunc, Thermo Fisher Scientific) were fixed in $4 \%$ paraformaldehyde at room temperature for $30 \mathrm{~min}$. After washing with phosphate-buffered saline (PBS), 5\% goat serum was added to the cells at room temperature for $1 \mathrm{~h}$. Cells were subsequently incubated with primary antibodies at $4^{\circ} \mathrm{C}$ overnight. Antibodies used for embryonic stem cell marker identification were stage-specific embryonic antigen-4 (SSEA-4) and Oct-4 (Santa Cruz). For Oct-4 staining, cells were permeabilized by $0.1 \%$ Triton X-100 for $20 \mathrm{~min}$ at room temperature before antibody incubation. Primary signals were detected using tetramethyl rhodamine ISO-thiocyanate (TRITC)-conjugated goat secondary antibodies (Santa Cruz) at room temperature for $1 \mathrm{~h}$ in the dark. Finally, each well was washed with PBS, nuclei were highlighted with Hoechst 33342 (Molecular Probe/Invitrogen), and immunofluorescence was detected by fluorescent microscopy.

\section{hES cell proliferation}

hES cells were plated on Matrigel-coated 96-well plates. The CyQuant cell proliferation assay (Molecular Probes) was conducted using a microplate spectrofluorometer (Gemini EM) at 24-, 48-, and 72-h time points. Eight samples were assayed and averaged.

\section{Spontaneous differentiation of hES cells}

hES cells were detached enzymatically and washed as described above. The cells were resuspended in an embryoid body (EB) medium containing DMEM supplemented with $20 \%$ FBS (Hyclone), then plated on $100 \mathrm{~mm}$ ultra-low attachment tissue culture dishes (Corning). The medium was changed every 2-3 days. EBs at day 8 were transferred to gelatin-coated dishes for adhesive culture. At day 14, EBs were removed, pelleted, and frozen at $-80^{\circ} \mathrm{C}$ for further analysis.

\section{Teratoma formation}

To track teratoma formation in vivo, hES cells of the H9 cell line were stably transduced with a self-inactivating lentiviral vector carrying a human ubiquitin promoter driving firefly luciferase and enhanced green fluorescence protein (Fluc-eGFP) as previously described [27]. After selection for stable populations, $5 \times 10^{5}$ low (passage $35-45$ ) and $5 \times 10^{5}$ high (passage 120-130) Fluc-eGFP hES cells were suspended in $25 \mu \mathrm{L}$ PBS, mixed with an equal volume of Matrigel for injection into the subcutaneous regions of the backs of the animals $(n=4,2$ spots for each group per mouse). Eight weeks after transplantation, teratomas were harvested and weighed. Cell differentiation was assayed by histological analysis and reverse transcriptasepolymerase chain reaction (RT-PCR). For histology, teratomas were fixed with $4 \%$ paraformaldehyde, and then embedded with paraffin, sectioned, and stained with hematoxylin and eosin. Light microscopy was used to observe the cells.

\section{Measurement of teratoma growth via bioluminescence imaging}

Cell signal was measured from day 2 after transplantation on a weekly basis for 8 weeks using a Xenogen IVIS 200 system (www.caliperls.com) as previously described [28]. After intraperitoneal injections of reporter probe D-Luciferin ( $375 \mathrm{mg} / \mathrm{kg}$ body weight), animals were imaged for a duration of $1 \mathrm{~s}$ to $1 \mathrm{~min}$. Imaging signals were quantified in units of maximum photons per second per square centimeter per steradian $\left(\mathrm{p} / \mathrm{s} / \mathrm{cm}^{2} / \mathrm{sr}\right)$.

\section{Reverse transcriptase-polymerase chain reaction}

Tissue samples were homogenized in Trizol (Invitrogen). Total RNA was isolated from cells using the RNeasy kit from Qiagen according to the manufacturer's instructions. cDNAs were obtained using $1 \mu \mathrm{g}$ RNA with an iScript cDNA synthesis kit (Bio-Rad). PCRs were carried out with $2 \mu \mathrm{L}$ cDNA template. The specific primers and reaction conditions are listed in Table 1.

\section{Measurement of intracellular reactive oxygen species}

DCFH-DA (Invitrogen) was used for reactive oxygen species (ROS) detection. When oxidized by ROS intracellularly, the nonfluorescent compound will become fluorescent. Cells from replicate cultures were dissociated with $0.5 \mathrm{mM}$ ethylenediaminetetraacetic acid (EDTA) to make a single cell suspension, and resuspended in DMEM/F12 medium containing $10 \mu \mathrm{M}$ DCFH-DA to a final density at $10^{6} \mathrm{cell} / \mathrm{mL}$. Cells were incubated at $37^{\circ} \mathrm{C}$ for $20 \mathrm{~min}$, washed once, resuspended in PBS, and kept on ice for an immediate detection by FACSCalibur (Becton, Dickinson Biosciences). Average measurements from 4 replicates were quantified as mean fluorescence intensity (MFI) $/ 10^{5}$ cells. 
Table 1. Primers and Reaction Conditions for Reverse Transcriptase-Polymerase Chain Reaction

\begin{tabular}{|c|c|c|c|c|}
\hline Gene & Primers & $\begin{array}{c}\text { Annealing } \\
\text { temperature }\left({ }^{\circ} \mathrm{C}\right)\end{array}$ & Cycles & Product (bp) \\
\hline \multirow[t]{2}{*}{ GAPDH } & 5'-ACСССТTCATTGACCTCAA-3' & \multirow[t]{2}{*}{54} & \multirow[t]{2}{*}{30} & \multirow[t]{2}{*}{436} \\
\hline & 5'-GCATGGACTGTGGTCATGAGT-3' & & & \\
\hline \multirow[t]{2}{*}{ Oct 4} & 5'-CTCACTCGGTTCTCGATACTGGTT-3' & \multirow[t]{2}{*}{58} & \multirow[t]{2}{*}{35} & \multirow[t]{2}{*}{198} \\
\hline & 5'-GGAAGGTATTCAGCCAAACGACCA-3' & & & \\
\hline \multirow[t]{2}{*}{ Flk-1 } & 5'-CCAGCCAAGCTGTCTCAGT-3' & \multirow[t]{2}{*}{61} & \multirow[t]{2}{*}{35} & \multirow[t]{2}{*}{272} \\
\hline & 5'-CTGCATGTCAGGTTGCAAAG-3' & & & \\
\hline \multirow[t]{2}{*}{ AFP } & 5'-GCTGGATTGTCTGCAGGATGGGGAA-3' & \multirow{2}{*}{60} & \multirow[t]{2}{*}{35} & \multirow{2}{*}{216} \\
\hline & 5'-TCCCCTGAAGAAAATTGGTTAAAAT-3' & & & \\
\hline \multirow[t]{2}{*}{ Nanog } & 5'-CCATTGCTATTCTTCGGCCAGTTG-3' & \multirow{2}{*}{58} & \multirow[t]{2}{*}{35} & \multirow[t]{2}{*}{176} \\
\hline & 5'-ACCAGAACTGTGTTCTCTTCCACC-3' & & & \\
\hline \multirow{2}{*}{$\operatorname{Rex} 1$} & 5'-ATCCTAAACAGCTCGCAGAAT-3' & \multirow{2}{*}{56} & \multirow[t]{2}{*}{35} & \multirow[t]{2}{*}{306} \\
\hline & 5'-GCGTACGCAAATTAAAGTCCAGA-3' & & & \\
\hline \multirow[t]{2}{*}{ Ncam } & 5'-GCCAGGAGACAGAAACGAAG-3' & \multirow[t]{2}{*}{60} & \multirow[t]{2}{*}{32} & \multirow[t]{2}{*}{164} \\
\hline & 5'-GGTGTTGGAAATGCTCTGGT-3' & & & \\
\hline \multirow[t]{2}{*}{ NeuroD } & 5'-AAGCCATGAACGCAGAGGAGGACT-3' & \multirow[t]{2}{*}{56} & \multirow[t]{2}{*}{32} & 578 \\
\hline & 5'-AGCTGTCCATGGTACCGTAA-3' & & & \\
\hline RunX2 & $5^{\prime}$-TTACTTACACCCCGCCAGTC- $3^{\prime}$ & 52 & 37 & 375 \\
\hline & $5^{\prime}$-TTCAATATGGTCGCCAAACA-3' & & & \\
\hline HNF4A & $5^{\prime}$-CTTCCTTCTTCATGCCAG-3' & 50 & 37 & 270 \\
\hline & 5'-ACACGTCCCCATCTGAAG-3' & & & \\
\hline Nkx2.5 & 5'-AGATCTTGACCTGCGTGGAC-3' & 57 & 37 & 224 \\
\hline & 5'-CTTCAAGCCAGAGGCCTACG-3' & & & \\
\hline Sox17 & 5'-GGGATACGCCAGTGACGACC-3' & 60 & 37 & 350 \\
\hline & $5^{\prime}$-GCTCTGCCTCCTCCACGAAG-3' & & & \\
\hline Albumin & $5^{\prime}$-AAGCAAAAAGAATGCCCT-3' & 50 & 37 & 290 \\
\hline & $5^{\prime}$-CTTGGGCTTGTGTTTCAC- $3^{\prime}$ & & & \\
\hline Glut2 & 5'-ATGCTCTGGTCCCTGTCTGTATCC-3' & 57 & 37 & 350 \\
\hline & 5'-TGACTAATAAGAATGCCCGTGACG-3' & & & \\
\hline Insulin & $5^{\prime}$-CAGGCTTTGTGAACCAACAC-3' & 55 & 37 & 327 \\
\hline & 5'-GCTTTATTCCATCTCTCTCTCGG-3' & & & \\
\hline
\end{tabular}

\section{Assessment of mitochondrial membrane potential}

Changes in mitochondrial membrane potential were estimated using the cationic fluorescent dye (JC-1; Molecular Probes) according to the manufacturer's instructions. Briefly, $5 \times 10^{5}$ cells were dissociated with $0.5 \mathrm{mM}$ EDTA and resuspended in $1 \mathrm{~mL}$ fresh complete medium as a single-cell suspension. The cell suspension was incubated with JC-1 $(2.5 \mu \mathrm{M})$ for $30 \mathrm{~min}$ at $37^{\circ} \mathrm{C}$ in the dark, followed by washing with PBS. Cells were analyzed using a flow cytometer equipped with a $488 \mathrm{~nm}$ argon laser (BD FACSCalibur). JC-1 is a dual-emission potential-sensitive probe that localizes to different sides of the mitochondrial membrane after cellular uptake. The ratio of red to green fluorescence from JC-1 can be quantified using flow cytometry and used as a measure of mitochondrial membrane potential. Cells treated with JC-1 and carbonyl cyanide 3-chlorophenylhydrazone, a potent uncoupler of oxidative phosphorylation, served as controls of dissipation of mitochondrial membrane potential.

\section{Measurement of oxygen consumption}

Cells from replicate cultures were dissociated with $0.5 \mathrm{mM}$ EDTA to make a single-cell suspension, and resuspended in mTeSR medium for immediate polarographic measurement of oxygen consumption using a Clark-type oxygen electrode (Hansatech) at $37^{\circ} \mathrm{C}$. mTeSR was used as the background oxygen value. This number was subtracted from the final oxygen consumption values obtained. Cells were maintained during the measurements at $37^{\circ} \mathrm{C}$ in a temperature-jacketed chamber, and oxygen consumption was monitored for $10 \mathrm{~min}$. Measurements with potassium cyanide were also performed as controls to ensure that the oxygen consumption observed was related to mitochondrial oxygen consumption. Average measurements from 4 replicates were quanitified as $\mathrm{nM}$ oxygen consumed $/ \mathrm{min} / 10^{6}$ cells.

\section{Fluorescent staining of mitochondria}

Mitochondria were stained with MitoTracker Green FM (Invitrogen), which preferentially accumulates in the mitochondrial matrix irrespective of changes in membrane potential. Cells were plated into chamber slides 1 day before staining. Adherent cells were exposed for $45 \mathrm{~min}$ to a $200 \mathrm{nM}$ MitoTracker Green FM solution at $37^{\circ} \mathrm{C}$ together with Hoechst 33342 nuclei staining. The resulting fluorescent signal was imaged with a laser-scanning confocal microscope (talamasca LSM510; Carl Zeiss). Mitochondrial volume was quantified using Volocity software (www.improvision .com). 3D mitochondrial imaging was reconstructed from a z-stack of optical sections. 3D image analysis tool from the software was used to quantify volume of mitochondria and expressed in $\mu \mathrm{m}^{3}$.

\section{hES cell karyotyping}

$\mathrm{hES}$ cells growing in $\log$ phase were treated with $0.1 \mathrm{mg} / \mathrm{mL}$ of colcemid for induction of mitotic arrest. Cell cultures were 
subsequently harvested by standard cytogenetic methods of trypsin dispersal, hypotonic shock, and fixed with 3:1 methanol:acetic acid [29]. Mitotic cell slide preparations were analyzed by the G-banding method and interpreted by an investigator blinded to study conditions [30].

\section{Detection of telomerase activity}

Telomerase activity was assayed in triplicates using the TRAPeze ELISA Detection Kit (Chemicon/Millipore) as per the manufacturer's instructions. Briefly, cells were lysed in CHAPS lysis buffer, and the cell extracts were frozen on dry ice. Telomerase was allowed to add telomeric repeats (GGTTAG) onto the $3^{\prime}$ end of a biotinylated telomerase substrate oligonucleotide at $30^{\circ} \mathrm{C}$ for $30 \mathrm{~min}$. The extended products were amplified by PCR using biotinylated telomerase substrate oligonucleotide and reverse primers and a deoxynucleotide mix containing dinitrophenyl-labeled deoxy-cytidine triphosphate (dCTP). The labeled PCR products were immobilized on streptavidin-coated microtiter plates and detected by an anti-dinitrophenyl antibody conjugated to horseradish peroxidase (HRP). The amount of product was determined by HRP activity using the HRP substrate 3,3',5,5'-tetramethylbenzidine and subsequent color development. The absorbance of the samples was measured at 450 and $690 \mathrm{~nm}$ with an automatic microplate reader (Multiskan EX; Thermo Scientific). Telomerase activity was determined using the following equation: absorbance at $450 \mathrm{~nm}-690 \mathrm{~nm}$. Lysis buffer alone and heat-inactivated lysed cells were used as negative controls. Heat inactivation was conducted by incubating cells at $85^{\circ} \mathrm{C}$ for $10 \mathrm{~min}$.

\section{Statistical analysis}

The data are represented as average \pm standard error of the mean and analyzed for statistical significance $(P<0.05)$ using 1 -way analysis of variance with the Bonferonni correction.

\section{Results}

\section{Morphology and cell marker expression over long-term culture}

hES cells maintained on feeder layers or in feeder-free culture grew as colonies of undifferentiated cells. No morphological changes were observed during the culture period of 82 passages (p38 to $\mathrm{p} 120$ ) for $\mathrm{H} 9$ or 62 passages (p61 to p123) for PKU1 cells. Immunostaining revealed retainment of stem cell surface markers (SSEA-4) and transcription factor expression (Oct4) from young to old passage cells (Fig. 1). FACS analysis was used to further quantify SSEA-4 surface marker expression on both young and old cells of the $\mathrm{H} 9$ and PKU1 cell lines. The percentage of hES cells found to stain positive for SSEA-4 did not significantly differ between young and old cells of either the H9 (p48: 97.1\% \pm 1.4 vs. p102: $98.0 \% \pm 0.8$ ) or PKU1 (p81: $69.2 \% \pm 2.0$ vs. p123: $57.5 \% \pm 6.4$ ) cell lines.

\section{In vitro hES cell proliferation and differentiation}

To determine whether long-term culture had any impact on hES cell proliferation, we used a CyQuant cell proliferation assay to quantify cell division in low and high passage
H9 cells, PKU1 cells, and H9 cells stably transduced with a double fusion (eGFP-Fluc) reporter gene (H9DF) [27]. Although proliferation rates were similar between young and old H9 cells at 24 and $48 \mathrm{~h}$, older passage H9 cells began proliferating at an elevated rate beginning $72 \mathrm{~h}$ after the initiation of the assay. For the H9DF cell line, a higher proliferation rate for older passage cells was observed at $48 \mathrm{~h}$, but led to reduced expansion at $72 \mathrm{~h}$ due to overconfluency. Increased proliferation was also detected in older passage PKU1 cells as compared with younger passage cells (Fig. 2AC). To compare the capacity of young and old hES cells to differentiate into derivatives of all 3 germ layers, EBs were formed from low and high passage cells in vitro. EBs were dissociated into pellets at day 14 and analyzed by semiquantitative RT-PCR to detect mRNA expression for pluripotency markers (Oct-4), ectoderm (Ncam), mesoderm (Flk-1), and endoderm $(A F P)$. Low and high passage $\mathrm{H} 9$ derivatives cultured under the same differentiation conditions expressed no significant differences at the mRNA level after EB formation (Fig. 2D). However, older passage PKU1 cells appeared to yield EBs that differentiated less robustly as detected by semiquantitative RT-PCR for the expression of Ncam, Flk-1, and AFP (Fig. 2E).

\section{In vivo teratoma formation by hES cells}

Teratoma formation upon transplantation into immunodeficient animals is a hallmark of hES cells [1]. To confirm whether high passage cells would retain the capacity to form derivatives of all 3 germ layers in vivo, we transplanted $5 \times 10^{5}$ low (p49) and $5 \times 10^{5}$ high (p126) passage H9DF cells into the subcutaneous regions of the backs of SCID mice ( $n=4$, two spots for each group per mouse). H9DF cells were stably transduced with a reporter gene expressing enhanced green fluorescent protein and firefly luciferase (eGFP-FLuc) for noninvasive tracking of proliferation by bioluminescence imaging [27]. No significant differences between growth rates of young and old passage cells were observed during the 8-week period (Fig. 3A). All mice developed teratomas, which were extracted 8 weeks after cell transplantation. Teratomas from the old passage cells weighed slightly more $(0.16 \pm 0.08 \mathrm{gm})$ than those arising from young passage cells $(0.15 \pm 0.08 \mathrm{gm})$, but this difference was not statistically significant $(P=0.83)$ (Fig. 3B). Hematoxylin and eosin staining of tumor samples revealed fairly similar visual patterns of differentiation between the 2 groups. Teratomas formed from young and old passage cells contained derivatives of all 3 germ layers that were easily identifiable via light microscopy (Fig. 3C). To quantify differentiation, semiquantitative RT-PCR was performed on the RNA of explanted tumors for pluripotency markers (Nanog, Oct4, and Rex1), ectodermal markers (Ncam and NeuroD), mesodermal markers (Runx2, HNF4a, and Nkx2.5), and endodermal markers (Sox17, Albumin, Glut2, and Insulin). Gene expression was normalized to the expression of a house keeping gene, GAPDH (Fig. 3D). Compared with low passage cells, teratomas arising from old passage cells were found to have depressed levels of expression for markers of endodermal lineage (Sox17 and Glut2), and elevated levels of mRNA specific to ectodermal lineage (neuroD) $(P<0.05)$. Older passage cells were also characterized by slightly elevated expression levels for markers of undifferentiation (Nanog and Rex1), and 

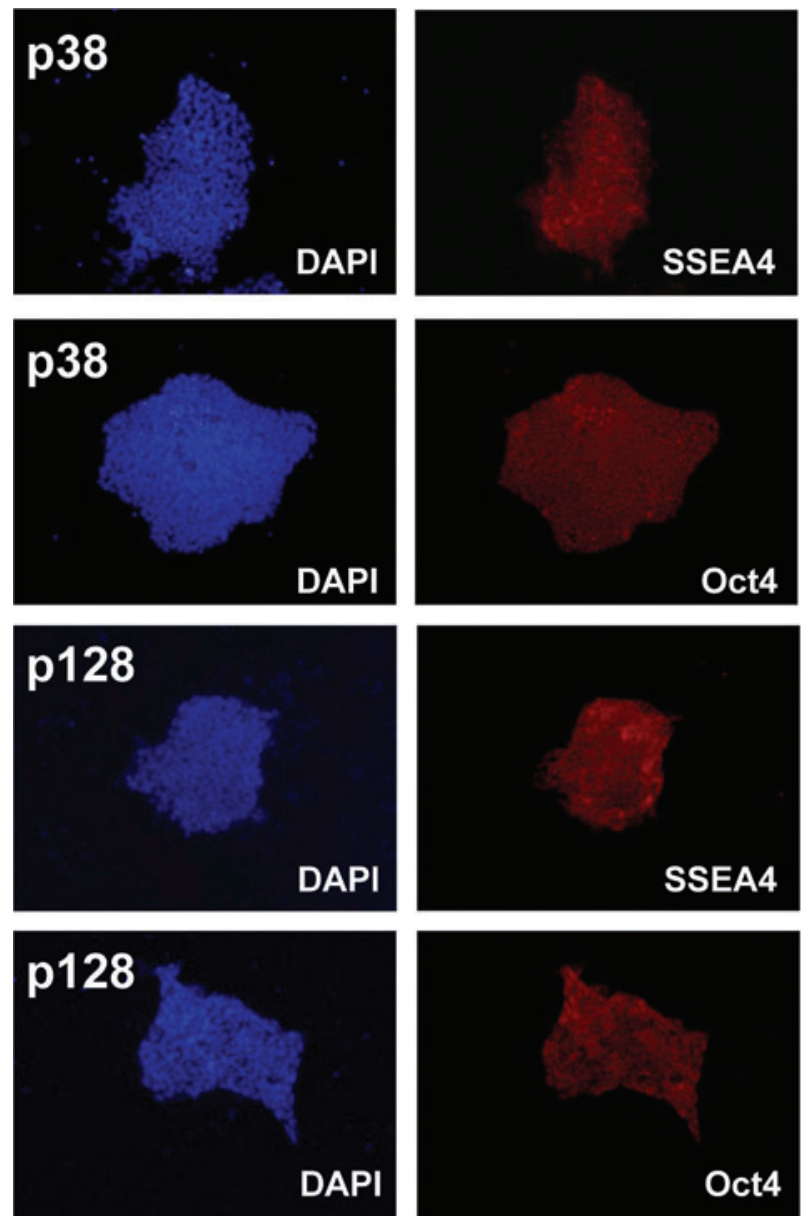
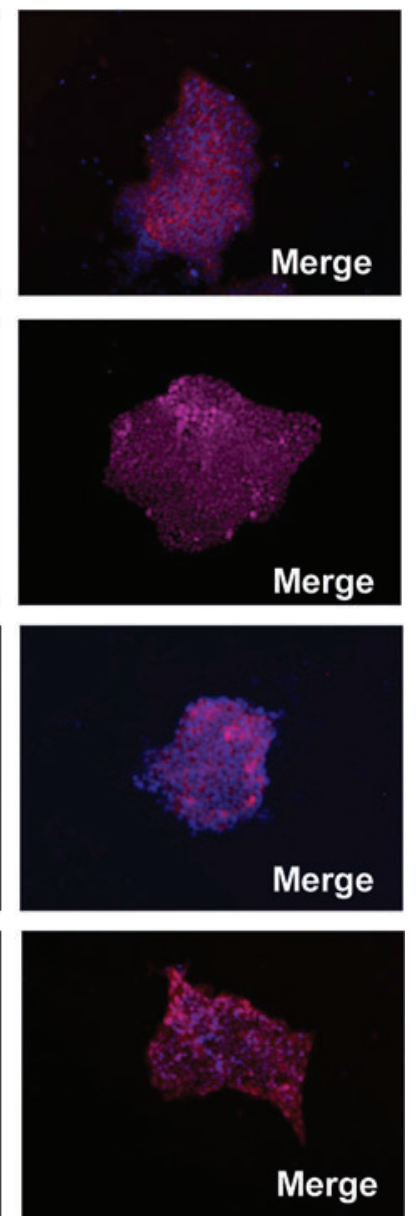

FIG. 1. hES cells can be maintained in an undifferentiated state over prolonged in vitro culture. hES cells (H9 line) were cultured for 90 passages on mouse embryonic fibroblast feeder layers (from passage 38 to 128). Expression of the pluripotency transcription factor Oct4 and cell surface marker SSEA-4 was examined by immunofluorescent staining (red). Nuclei were costained with DAPI (blue). Late passage cells were not significantly different from younger passage cells in cell morphology or expression of pluripotency markers. DAPI, 4',6-diamidino-2phenylindole; hES cells, human embryonic stem cells; SSEA, stagespecific embryonic antigen. depressed levels of mRNA specific to mesodermal lineages (Runx2), although these differences were not statistically significant.

\section{Mitochondrial function of young versus old hES cells}

Although a causal relationship between abnormalities in mitochondria and premature aging has only been established in recent years [31], it is commonly accepted within the scientific community that mitochondrial dysfunction is a marker of senescence [32]. To investigate whether mitochondrial function is impaired by prolonged passage, we compared several parameters of mitochondrial function in young and old hES cells. Functional analysis was determined by the measurement of intracellular ROS, oxygen consumption, and mitochondrial membrane potential. Mitochondrial volume within the cell was also assessed using confocal microscopy. Late passage $\mathrm{H} 9$ cells were found to have higher levels of intracellular ROS $\left(1,071 \pm 23 \mathrm{MFI} / 10^{5}\right.$ cells $)$ than younger passage cells $\left(847 \pm 147 \mathrm{MFI} / 10^{5}\right.$ cells, $P<0.05$, Fig. $\left.4 \mathrm{~A}\right)$. Late passage PKU1 cells were also observed to have elevated levels of ROS $\left(2,117 \pm 150 \mathrm{MFI} / 10^{5}\right.$ cells) compared with young passage cells $\left(1,758 \pm 151 \mathrm{MFI} / 10^{5}\right.$ cells) (Fig. 4B). Older passage $\mathrm{H} 9$ cells were also observed to have a slightly decreased rate of oxygen consumption (3.38 $\pm 0.69 \mathrm{nmol}$ $\mathrm{O}_{2} / \mathrm{min} / 10^{6}$ cells) compared with young cells $(4.04 \pm 0.47$ $\mathrm{nmol} \mathrm{O} 2 / \mathrm{min} / 10^{6}$ cells) (Fig. $4 \mathrm{C}$ ).
Mitochondrial membrane potential $(\Delta \Psi \mathrm{m})$ is a measure of the transmembrane electrical gradient of mitochondria. We measured $\Delta \Psi \mathrm{m}$ in low and high passage hES cells using a JC-1 assay kit. $\Delta \Psi \mathrm{m}$ was numerically calculated as the ratio between the intramitochondrial aggregate (red) signal to cytoplasmic monomeric (green) signal of the dyes [33]. Late passage H9 cells were found to have significantly elevated $\Delta \Psi \mathrm{m}(34.38 \pm 8.70)$ when compared with young passage cells $(12.97 \pm 2.92)(P<0.001$, Fig. 4D). Similar findings were observed in old passage PKU1 cells $(2.33 \pm 0.05)$ compared with younger passage cells $(1.87 \pm 0.14)(P<0.01$, Fig. $4 \mathrm{E})$.

To observe the mitochondria and evaluate mitochondrial mass using confocal fluorescence microscopy, we used a MitoTracker $^{\circledR}$ mitochondrion-selective probe assay. The total mitochondrial volume in low and high passage hES cells was documented (Fig. 4F). In young passage H9 cells, the total volume of mitochondria was on average, $220 \pm 106 \mu \mathrm{m}^{3} /$ cell. In contrast, older cells displayed a much higher mean volume of $528 \pm 81 \mathrm{~mm}^{3} /$ cell $(P<0.01$, Fig. $4 \mathrm{G})$. Mitochondria volume of young passage PKU1 cells was $390 \pm 143 \mu \mathrm{m}^{3} /$ cell, and increased to $990 \pm 209 \mu^{3} /$ cell for old passage cells $(P<0.005$, Fig. 4H)

\section{Karyotypic analysis of hES cells}

Karyotyping is a traditional measure of cell line stability and safety [34]. To confirm that hES cells maintained 

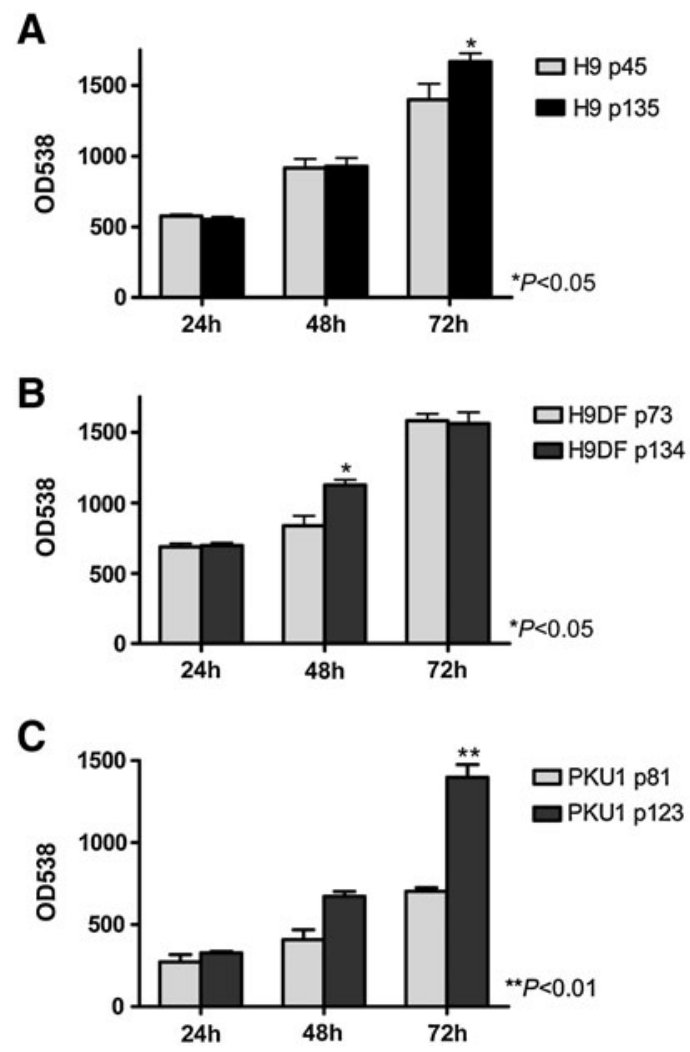
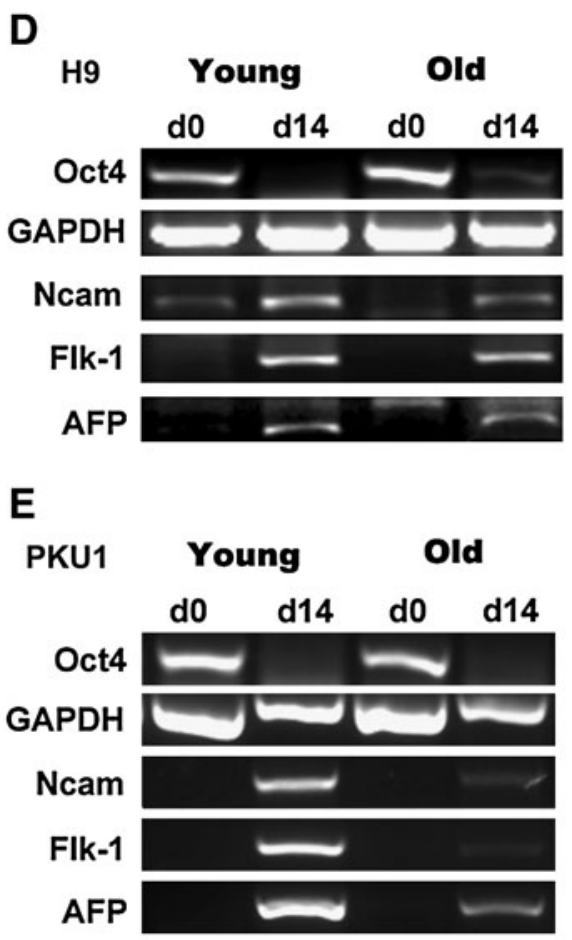

FIG. 2. In vitro hES cell proliferation and differentiation. (A-C) Proliferation of young and old passage H9, H9DF, and PKU1 hES cells was quantified at 24,48 , and $72 \mathrm{~h}$ by a CyQuant cell proliferation assay. Late passage hES cells were generally found to have a higher proliferation rate compared with younger passage cells. Specifically, older passage H9 cells exhibited elevated proliferation a $72 \mathrm{~h}, \mathrm{H} 9 \mathrm{DF}$ cells at $48 \mathrm{~h}$, and PKU1 cells at 48 and $72 \mathrm{~h}$ compared with younger counterparts. (D, E) Young and old passage cells were allowed to spontaneously differentiate into embroid bodies in vitro, after which cell derivatives of all 3 germ layers were examined by semiquantitative reverse transcriptase-polymerase chain reaction. H9 cells exhibited no significant differences in the expression patterns of endoderm marker (AFP), mesoderm marker (Flk-1), and ectoderm marker (Ncam) between young and old passage cells. Older passage PKU1 cells exhibited reduced capacity to form cell derivatives of the 3 germ layers however.

chromosomal integrity over the period of this experiment, we karyotyped H9 and PKU1 hES cells by standard Gbanding techniques. For H9 cells, young passage cells (p48) presented with a normal female karyotype as 46, XX. Cells passaged to p120 were found to have a karyotype change event consisting of a translocation between the long arms of chromosomes 1 and 9, resulting in the deletion of one of the long arms of chromosome 9 and the duplication of one of the long arms of chromosome 1 . The new karyotype presented itself as 46, XX, der(9)t(1;9)(q31;q22). PKU1 cells were observed to have a stable karyotype for the culture period of 1 year. Both early and late passage PKU1 cells had normal female karyotype as 46, XX (Fig. 5).

\section{Telomerase activity of hES cells}

High telomerase activity or expression of telomerase reverse transcriptase (TERT), the catalytic protein subunit of telomerase, is regarded as a marker of stem cells [35]. Telomere shortening, which occurs as TERT activity declines, is one of the fundamental molecular mechanisms underlying cell aging [36-38]. A telomere repeat amplification protocol assay was performed to compare telomerase activity in low (p39) and high passage (p110) H9 cells. Both young and old H9 cells showed equally elevated levels of telomerase activity $(5,014 \pm 294$ vs. $5,030 \pm 377$ unit $/ \mu g, P=$ NS, Fig. 6$)$. To compare these levels of enzyme activity with cells known to have upregulated levels of TERT, we also measured telomerase activity in 4 human cancer cell lines: (1) a human pancreatic cancer cell line BXPC3; (2) a human glioblastomaastrocytoma, epithelial-like cell line U87MG; (3) a human leukemic monocyte lymphoma cell line U937; and (4) a human colon adenocarcinoma cell line HT29. On average, telomerase activity in both young and old passage $\mathrm{H} 9 \mathrm{hES}$ cells was 2-fold higher than in tumor cell lines $(2,562 \pm 1,909$ unit $/ \mu \mathrm{g}$ ). Further, the level of telomerase activity in hES cells remained stable from passage 39 to passage 110 .

\section{Discussion}

hES cells are defined by their capacity for unlimited selfrenewal and pluripotency. However, the issue of whether hES cells can be maintained stably under prolonged in vitro conditions is still unresolved. In this study, the long-term stability of hES cells was confirmed by the preservation of stem cell surface marker expression, telomerase activity, and 
A

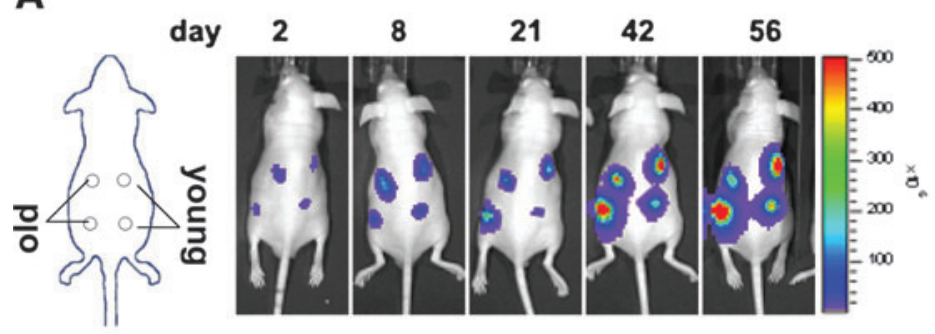

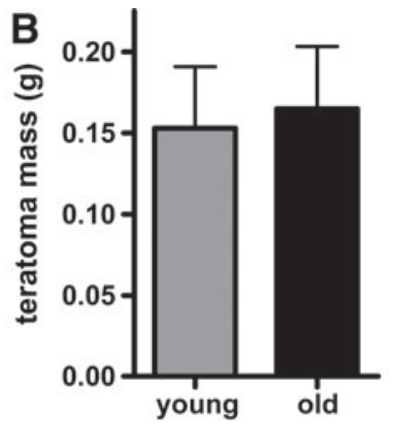

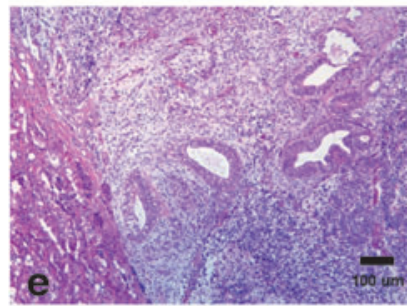

C

P49
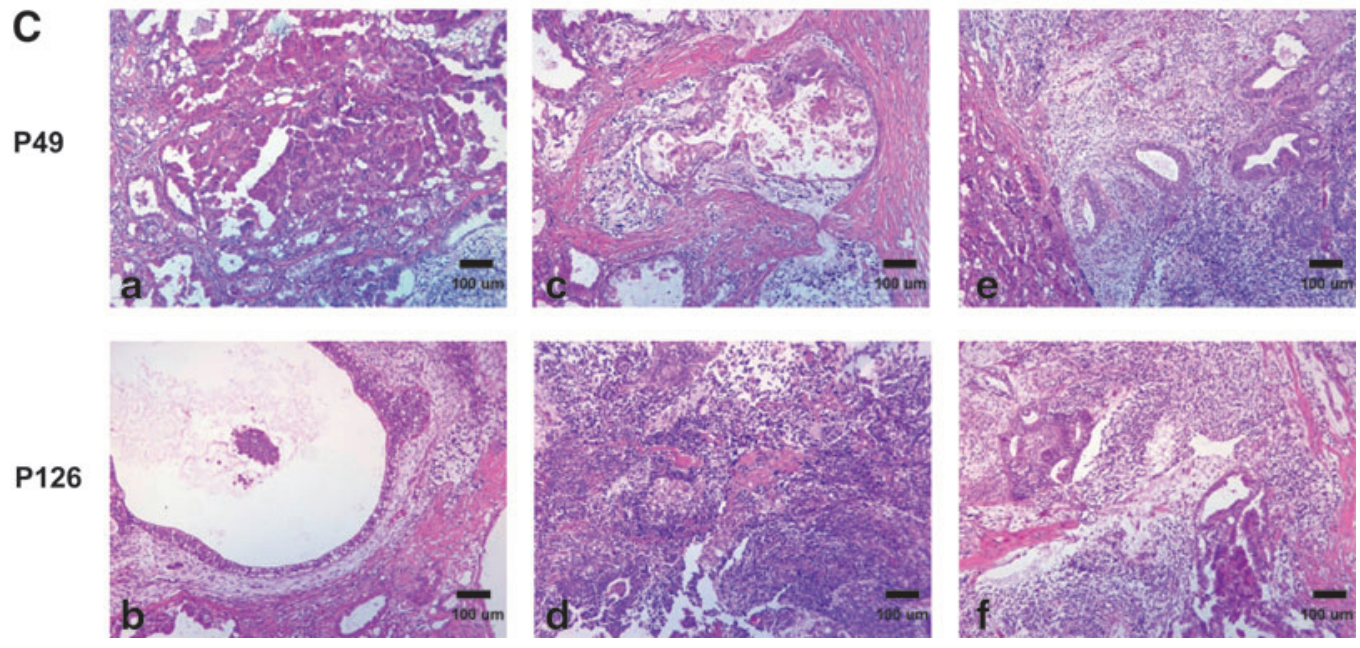

D

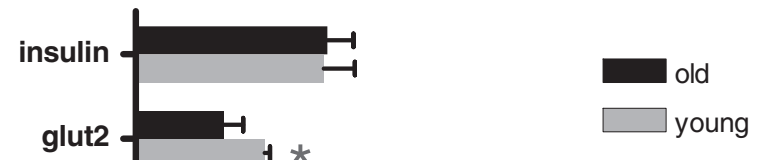

FIG. 3. Teratoma formation by early and late passage human ES cells. A half million H9 cells from early passage (P49) and late passage (P126) were injected subcutaneously on the backs of nude mice. Tetatomas were harvested 8 weeks after injection, weighed, and processed for hematoxylin and eosin histology. (A) Bioluminescence imaging of subcutaneous teratoma formation revealed no significant differences in growth between young and old passage hES cells implanted subcutaneously. (B) No significant difference in weight was observed between teratomas arising from young and late passage H9DF cells. (C) Postmortem histological analysis of transplanted hES cells showed (a) immature brain-like neural cell formation; (b) squamous cell differentiation with keratin pearls; (c, d) osteochondroid formation; (e, f) respiratory epithelium with ciliated columnar and mucin producing goblet cells. Both early and late passage hES cells can form all 3 germ layer morphologies. (D) Reverse transcriptase-polymerase chain reaction analysis of explanted teratomas at week 8. Gene expression was normalized to a house keeping gene, GAPDH. Both young and old passage hES cells formed derivatives of all 3 germ layers. Old passage hES cells were characterized by elevated levels of expression for ectodermal markers (NeuroD) and decreased levels of expression for endodermal markers (Sox17 and Glut2) as compared with younger passage counterparts. 
A

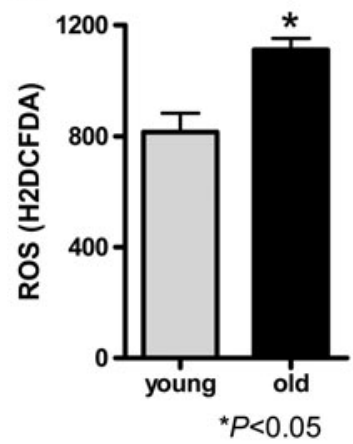

E

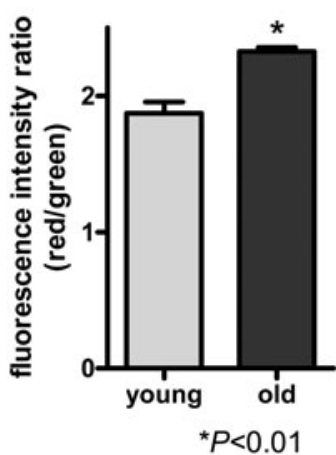

B

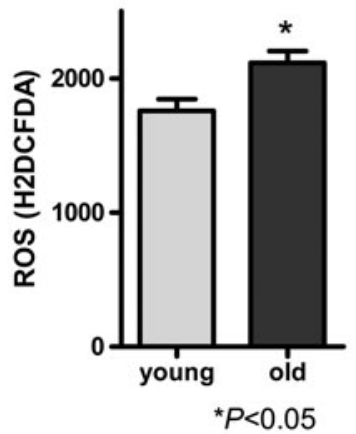

F

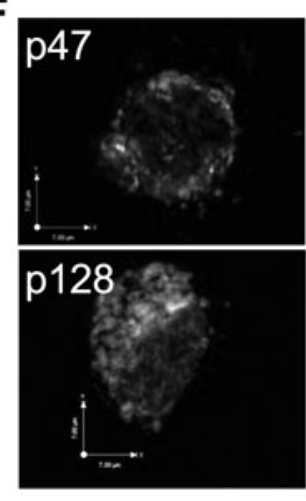

C

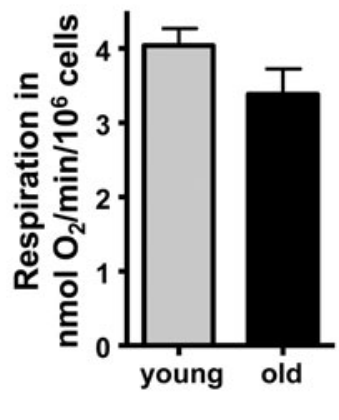

G

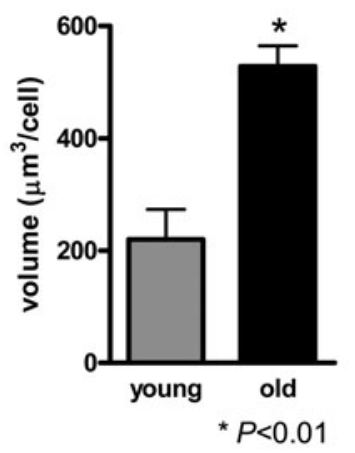

D

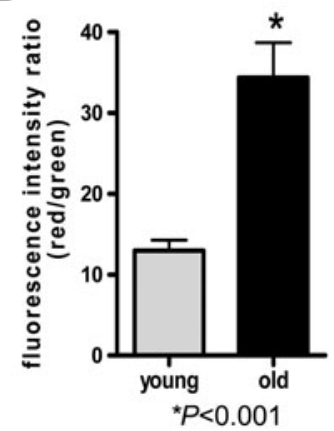

H

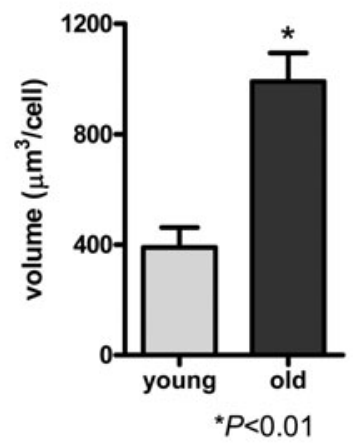

FIG. 4. Mitochondrial function of young versus old hES cells. hES cells undergoing prolonged cell culture were observed to have mitochondrial dysfunction. (A, B) Intracellular ROS were quantified in young and old passage hES cells by DCFH-DA fluorescent staining. Older passage cells were found to have higher ROS production than younger passage cells in both the H9 and PKU1 cell lines, suggesting that hES cells are susceptible to oxidative stress with extended culture. (A) H9 cells; (B) PKU1 cells. (C) Measurement of oxygen consumption revealed a decrease in $\mathrm{O} 2$ consumption in old passage $\mathrm{H} 9$ cells, but this result was not significant. (D, E) FACS analysis of MitoProbe JC-1 staining showed higher mitochondrial membrane potential in older passage H9 and PKU1 cells as compared with younger counterparts. (D) H9 cells; (E) PKU1 cells. (F) Confocal microscopy was used to assess mitochondrial volume in fluorescent images of living H9 and PKU1 cells stained with MitoTracker dye. (Scale bar: $7 \mu \mathrm{m})(\mathbf{G}, \mathbf{H})$ Total mitochondrial volume was quantified using confocal microcopy. Mitochondrial volume was found to be highly elevated in older passage hES cells as compared with younger passage cells. (G) H9 cells; (H) PKU1 cells. ROS, reactive oxygen species.

capacity to differentiate into derivatives of all 3 germ layers upon EB formation in vitro and teratoma formation in vivo. Karyotyping and analysis of mitochondrial function, however, revealed differences between older and younger passage cells. The karyotype abnormalities $[46, X X$, der $(9) t(1 ; 9)$ (q31;q22)] observed in H9 cells suggest prolonged culture of $\mathrm{hES}$ cells or their derivatives may result in heightened risk of cancerous transformation or functional abnormalities. While late passage $\mathrm{H} 9$ cells were observed to have heightened cell proliferation rates as compared with younger passage cells, such increases in cell division were also found in the PKU1 cell line which possessed a normal karyotype. This suggests that increases in cell proliferation for older passage hES cells are not necessarily the result of a karyotypic abnormalities. A recent study by Park et al. shows that correlations may exist between increases in in vitro cellular division and tumorigenesis [39]. However, in this study in vivo bioluminescence imaging of teratoma formation did not reveal any significant differences between tumor formation of young and old hES cells.

Interestingly, our examination of teratomas by semiquantitative PCR revealed that the age of hES cells may af- fect their proclivity for differentiation into specific germ lineages. Younger passage H9 cells differentiated evenly into derivatives of ectodermal, mesodermal, and endodermal germ layers. In contrast, higher passage hES cells primarily formed derivatives of ectodermal lineage and were characterized by lower levels of mRNA for derivatives of endodermal origin. Preferential differentiation into ectodermal derivatives was most likely influenced by the presence of basic fibroblast growth factor in the culture media for the duration of the experiment. Basic fibroblast growth factor has been shown to be a strong neurotrophic factor and can be used to induce differentiation of hES cells into neuroectoderm [40]. These results indicate that both the culture medium and cell age may potentially influence the preferential differentiation of hES cells during prolonged in vitro culture. Further studies will be required to examine the extent to which prolonged cell culture affects the use of high passage hES cells for derivation of terminally differentiated cells in models of cell replacement therapy.

Mitochondria were first implicated in the aging process for over 50 years ago by Denham Harman in his free radical 


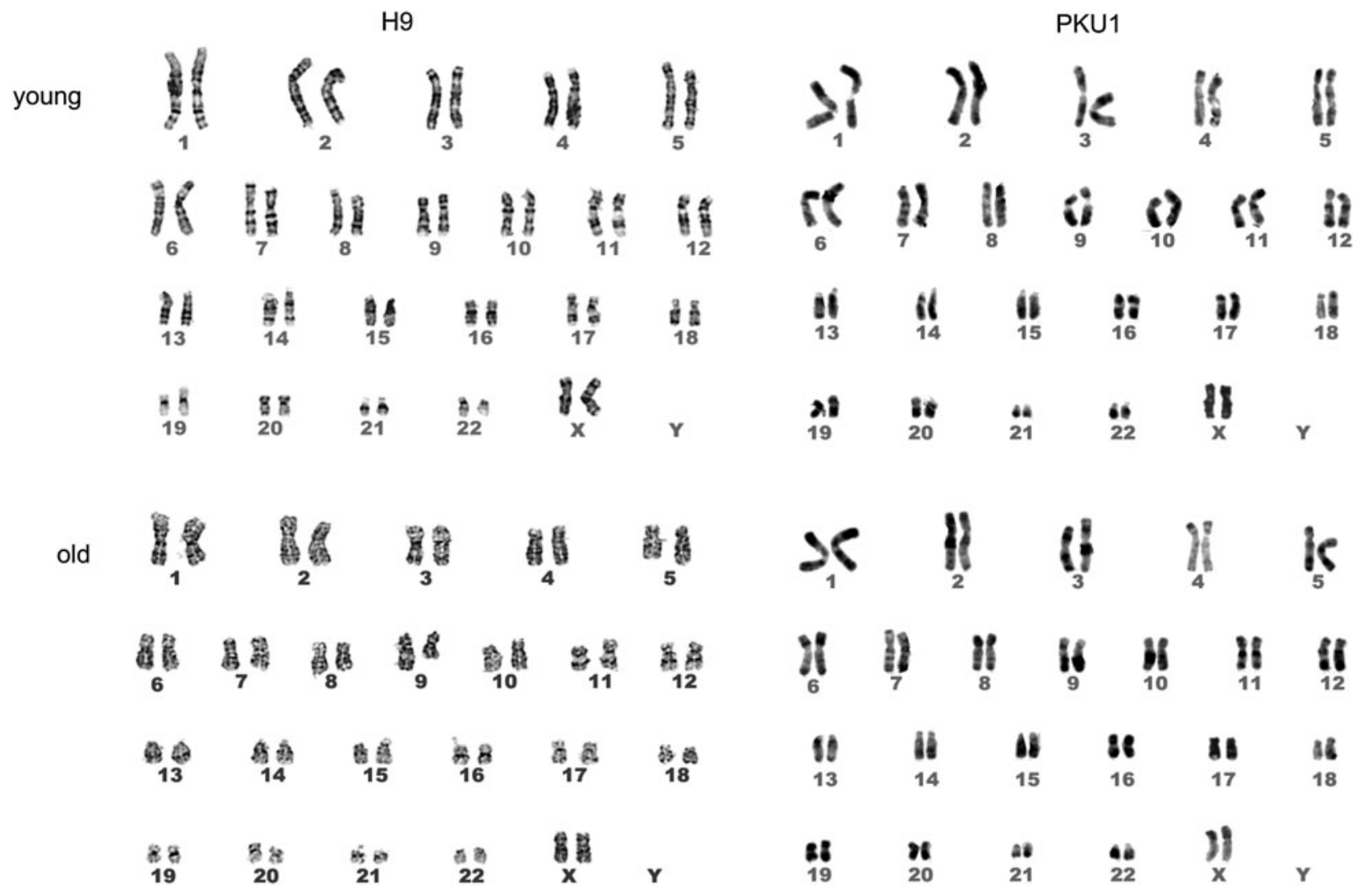

FIG. 5. Karyotyping of early and late passage hES cells. Passage $48 \mathrm{H} 9$ cells showed a normal female karyotype as 46 , XX. However, passage $120 \mathrm{H} 9$ cells were found to have an unbalanced chromosomal translocation involving the long arms of chromosomes nos. 1 and 9, resulting in the karyotypic change 46,XX,der(9)t(1;9)(q31;q22). Both passage 82 and 124 PKU1 cells retained normal female karyotype as $46, \mathrm{XX}$.

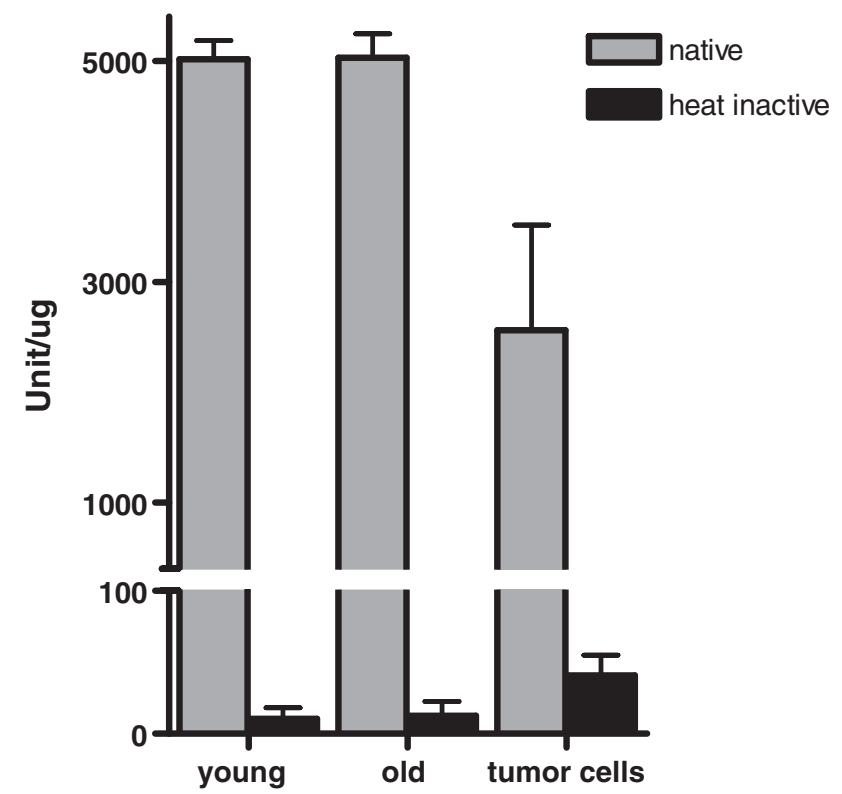

FIG. 6. Detection of telomerase activity by telomere repeat amplification protocol assay. Both early and late passage $\mathrm{H} 9$ hES cells maintained high levels of telomerase activity as compared with tumor cells (averaged values for BXPC3, U87MG, U937, and HT29). There was no significant difference found between young and old passage hES cells $(5014 \pm 294$ unit/ $\mu \mathrm{g}$ for young passage cells versus $5030 \pm 377 \mathrm{unit} / \mu \mathrm{g}$ for old passage cells, $P=\mathrm{NS}$ ). theory of aging [41]. In his original article, Harman postulated that ROS made as a byproduct of oxidative phosphorylation drive the aging process via free radical damage to mitochondrial DNA (mtDNA), which in turn leads to decreased mitochondrial function and the release of proapoptotic factors such as cytochrome C. A large body of evidence has since validated this theory [32], and recently several articles have shown a causal relationship between mitochondrial defects and premature aging [31,42]. Declines in mitochondrial function are commonly observed with aging in somatic cells [43]. Despite these findings, little research has been conducted on changes that occur in the mitochondria of ES cells during prolonged culture.

To investigate whether functional changes occurred in the mitochondria of hES cells over time, we compared ROS content, mitochondrial membrane potential, and oxygen consumption in young and old passage hES cells. The results from our mitochondrial experiments support the idea that hES cells maintained in culture are not totally immune to the effects of senescence. A number of studies have tracked changes in the mitochondria of hES cells that occur with aging and development. These studies have shown that not only does the intracellular localization of mitochondria change with blastocyst development, but also the differentiation of hES cells is associated with increases in mitochondrial mass, ATP production, and ROS production [44]. Schieke et al. recently found that when mouse ES cells are separated by mitochondrial membrane potential, 
different groups showed distinct germ layer differentiation tendency and teratoma formation rate [45]. Our results are highly similar to these reports, in that we found older passage hES cells to have an increased mitochondrial mass, a higher ROS content, and an elevated mitochondrial membrane potential compared with their younger passage counterparts.

Senescent mitochondria can affect clinical use of hES cells in several ways. In somatic cells, defects in mitochondria that occur with aging have been shown to impair ATP production and cause early cellular senescence [46,47]. Acceleration in mitochondrial proliferation and development has also been correlated to a loss of pluripotency. Two recent reports have shown that in the developing blastocyst, elevation in mtDNA copy number and increased drive for aerobic respiration correspond to an ensuing loss of pluripotency $[48,49]$. Similarly, repression of mitochondrial development by placing hES cells in hypoxic conditions has been shown to slow differentiation [50]. In this report, the elevated volume of mitochondria observed in older passage hES cells by confocal microscopy suggests elevated proliferation and expansion of mitochondrial number. This may indicate impaired removal of damaged and nonfunctional mitochondria in older cells, and/or fusion of existing mitochondria as a compensatory mechanism against the observed mitochondrial dysfunction. This may potentially result in the accumulation of ROS, which would explain the increased ROS content observed in higher passage cells.

Finally, mitochondrial membrane potential is a pivotal controller of respiratory rate, ATP synthesis, and the generation of ROS, and is itself controlled by electron transport and proton leaks. Although it is unclear what causes the increase of mitochondrial membrane potential in late passage hES cells, a high mitochondrial membrane potential in our results may be the cause for the observed increase in ROS production, as it has been clearly shown that at high $\Delta \Psi \mathrm{ms}$ even a small increase in membrane potential can give rise to a large stimulation of $\mathrm{H}_{2} \mathrm{O}_{2}$ production [51]. If older passage $\mathrm{hES}$ cells are susceptible to the generation of increased amounts of ROS due to changes in $\Delta \Psi \mathrm{m}$, the accumulation of oxidative damage over the course of prolonged culture may have serious implications for the use of late passage cells in therapeutic medicine.

In summary, we found that although hES cells are resistant to most aspects of senescence such as loss of pluripotency, telomerase activity, and stem cell marker expression, they are not completely immune to the aging process as their mitochondrial function declines, making hES cell mitochondria vulnerable to insults. Changes in mitochondrial function observed with prolonged culture are significant because of their effect upon cellular metabolism and their potential to adversely affect ROS generation and integrity of mtDNA. The nuclear genome may also be vulnerable to these insults as evidenced by a chromosomal translocation after 80 passages in $\mathrm{H} 9$ cells. Karyotypic abnormalities did not result in significant changes in teratoma formation upon transplantation into immunodeficient animals, but have the potential to place the cell at an increased risk for malignant transformation later. PKU1 cells cultured over 120 passages were not observed to have a karyotypic change, but exhibited similar differences in ROS production, mitochondrial membrane potential, and volume, suggesting that these changes result from prolonged cell culture and not chromosomal instability. Preferential differentiation of older passage hES cells in vivo into derivatives of ectodermal lineage is another issue that arises from long-term culture, but is most likely a result of the culture medium used to grow the cells. Taken together, this is the first study to conduct an in-depth functional evaluation of hES cells after long-term culture. This study adds valuable new knowledge regarding the little-understood senescence process of hES cells, as well as insights into how long-term passaged hES cells can be used for future clinical applications.

\section{Acknowledgments}

We are grateful to Andrew J. Connolly for assistance with histological analysis. We thank funding support from NIH DP2OD004437, AI085575, HL091453, HL089027 (J.C.W.), HHMI Research Fellowship (A.S.L.), and the National 973 project of China 2011CB964800 (T.C.).

\section{Author Disclosure Statement}

No competing financial interests exist.

\section{References}

1. Thomson JA, J Itskovitz-Eldor, SS Shapiro, MA Waknitz, JJ Swiergiel, VS Marshall and JM Jones. (1998). Embryonic stem cell lines derived from human blastocysts. Science 282:1145-1147.

2. Laflamme MA, KY Chen, AV Naumova, V Muskheli, JA Fugate, SK Dupras, H Reinecke, C Xu, M Hassanipour, S Police, O'Sullivan C, L Collins, Y Chen, E Minami, EA Gill, S Ueno, C Yuan, J Gold and CE Murry. (2007). Cardiomyocytes derived from human embryonic stem cells in prosurvival factors enhance function of infarcted rat hearts. Nat Biotechnol 25:1015-1024.

3. Cao F, RA Wagner, KD Wilson, X Xie, Fu J-D, M Drukker, A Lee, RA Li, SS Gambhir, IL Weissman, RC Robbins and JC Wu. (2008). Transcriptional and functional profiling of human embryonic stem cell-derived cardiomyocytes. PLoS ONE 3:e3474.

4. Ben-Hur T, M Idelson, H Khaner, M Pera, E Reinhartz, A Itzik and BE Reubinoff. (2004). Transplantation of human embryonic stem cell-derived neural progenitors improves behavioral deficit in Parkinsonian rats. Stem Cells 22:12461255.

5. Kim JH, JM Auerbach, JA Rodriguez-Gomez, I Velasco, D Gavin, N Lumelsky, SH Lee, J Nguyen, R Sanchez-Pernaute, K Bankiewicz and R McKay. (2002). Dopamine neurons derived from embryonic stem cells function in an animal model of Parkinson's disease. Nature 418:50-56.

6. Kroon E, LA Martinson, K Kadoya, AG Bang, OG Kelly, S Eliazer, H Young, M Richardson, NG Smart, J Cunningham, AD Agulnick, D'Amour KA, MK Carpenter and EE Baetge. (2008). Pancreatic endoderm derived from human embryonic stem cells generates glucose-responsive insulin-secreting cells in vivo. Nat Biotechnol 26:443-452.

7. Soto-Gutierrez A, N Kobayashi, JD Rivas-Carrillo, N Navarro-Alvarez, D Zhao, T Okitsu, $\mathrm{H}$ Noguchi, $\mathrm{H}$ Basma, $\mathrm{Y}$ Tabata, Y Chen, K Tanaka, M Narushima, A Miki, T Ueda, HS Jun, JW Yoon, J Lebkowski, N Tanaka and IJ Fox. (2006). Reversal of mouse hepatic failure using an implanted liverassist device containing ES cell-derived hepatocytes. Nat Biotechnol 24:1412-1419. 
8. Narayan AD, JL Chase, RL Lewis, X Tian, DS Kaufman, JA Thomson and ED Zanjani. (2006). Human embryonic stem cell-derived hematopoietic cells are capable of engrafting primary as well as secondary fetal sheep recipients. Blood 107:2180-2183.

9. Tian X, PS Woll, JK Morris, JL Linehan and DS Kaufman. (2006). Hematopoietic engraftment of human embryonic stem cell-derived cells is regulated by recipient innate immunity. Stem Cells 24:1370-1380.

10. Chen SL, WW Fang, F Ye, YH Liu, J Qian, SJ Shan, JJ Zhang, RZ Chunhua, LM Liao, S Lin and JP Sun. (2004). Effect on left ventricular function of intracoronary transplantation of autologous bone marrow mesenchymal stem cell in patients with acute myocardial infarction. Am J Cardiol 94:92-95.

11. Wollert KC, GP Meyer, J Lotz, S Ringes-Lichtenberg, P Lippolt, C Breidenbach, S Fichtner, T Korte, B Hornig, D Messinger, L Arseniev, B Hertenstein, A Ganser and $\mathrm{H}$ Drexler. (2004). Intracoronary autologous bone-marrow cell transfer after myocardial infarction: the BOOST randomised controlled clinical trial. Lancet. 364:141-148.

12. Goldstein S. (1990). Replicative senescence: the human fibroblast comes of age. Science 249:1129-1133.

13. Finkel T and NJ Holbrook. (2000). Oxidants, oxidative stress and the biology of ageing. Nature 408:239-247.

14. Kamminga LM and GD Haan. (2006). Cellular memory and hematopoietic stem cell aging. Stem Cells 24:1143-1149.

15. Cervantes RB, JR Stringer, C Shao, JA Tischfield and PJ Stambrook. (2002). Embryonic stem cells and somatic cells differ in mutation frequency and type. Proc Natl Acad Sci USA 99:3586-3590.

16. Amit M, MK Carpenter, MS Inokuma, C-P Chiu, CP Harris, MA Waknitz, J Itskovitz-Eldor and JA Thomson. (2000). Clonally derived human embryonic stem cell lines maintain pluripotency and proliferative potential for prolonged periods of culture. Dev Biol 227:271-278.

17. Rosler ES, GJ Fisk, X Ares, J Irving, T Miura, MS Rao and MK Carpenter. (2004). Long-term culture of human embryonic stem cells in feeder-free conditions. Dev Dyn 229: 259-274.

18. Brimble SN, X Zeng, DA Weiler, Y Luo, Y Liu, IG Lyons, WJ Freed, AJ Robins, MS Rao and TC Schulz. (2004). Karyotypic stability, genotyping, differentiation, feeder-free maintenance, and gene expression sampling in three human embryonic stem cell lines derived prior to August 9, 2001. Stem Cells Dev 13:585-597.

19. Buzzard JJ, NM Gough, JM Crook and A Colman. (2004). Karyotype of human ES cells during extended culture. Nat Biotech 22:381-382.

20. Rugg-Gunn PJ, AC Ferguson-Smith and RA Pedersen. (2005). Epigenetic status of human embryonic stem cells. Nat Genet 37:585-587.

21. Mitalipov SM. (2006). Genomic imprinting in primate embryos and embryonic stem cells. Reprod Fertil Dev 18: 817-821.

22. Sun BW, AC Yang, Y Feng, YJ Sun, Y Zhu, Y Zhang, H Jiang, CL Li, FR Gao, ZH Zhang, WC Wang, XY Kong, G Jin, SJ Fu and Jin Y. (2006). Temporal and parental-specific expression of imprinted genes in a newly derived Chinese human embryonic stem cell line and embryoid bodies. Hum Mol Genet 15:65-75.

23. Draper JS, K Smith, P Gokhale, HD Moore, E Maltby, J Johnson, L Meisner, TP Zwaka, JA Thomson and PW Andrews. (2004). Recurrent gain of chromosomes $17 \mathrm{q}$ and 12 in cultured human embryonic stem cells. Nat Biotechnol 22:53-54.
24. Maitra A, DE Arking, N Shivapurkar, M Ikeda, V Stastny, K Kassauei, G Sui, DJ Cutler, Y Liu, SN Brimble, K Noaksson, J Hyllner, TC Schulz, X Zeng, WJ Freed, J Crook, S Abraham, A Colman, P Sartipy, S Matsui, M Carpenter, AF Gazdar, M Rao and Chakravarti A. (2005). Genomic alterations in cultured human embryonic stem cells. Nat Genet 37:1099-1103.

25. Pannetier M and Feil R. (2007). Epigenetic stability of embryonic stem cells and developmental potential. Trends Biotechnol 25:556-562.

26. Peng H-m and G-a Chen. (2006). Serum-free medium cultivation to improve efficacy in establishment of human embryonic stem cell lines. Hum Reprod 21:217-222.

27. Li Z, Y Suzuki, M Huang, F Cao, X Xie, AJ Connolly, PC Yang and JC Wu. (2008). Comparison of reporter gene and iron particle labeling for tracking fate of human embryonic stem cells and differentiated endothelial cells in living subjects. Stem Cells 26:864-873.

28. Sun N, A Lee and JC Wu. (2009). Long term non-invasive imaging of embryonic stem cells using reporter genes. Nature protocols 4:1192-1201.

29. Barch MJ, T Knutsen and JL Spurbeck. (1997). The AGT Cytogenetics Laboratory Manual. Chapter 4 (3rd Edition). Lippincott-Raven, New York.

30. Seabright M. (1971). A rapid banding technique for human chromosomes. Lancet 2:971-972.

31. Vermulst M, J Wanagat, GC Kujoth, JH Bielas, PS Rabinovitch, TA Prolla and LA Loeb. (2008). DNA deletions and clonal mutations drive premature aging in mitochondrial mutator mice. Nat Genet 40:392-394.

32. Balaban RS, S Nemoto and T Finkel. (2005). Mitochondria, oxidants, and aging. Cell 120:483-495.

33. Smiley ST, M Reers, Mottola-Hartshorn C, M Lin, A Chen, TW Smith, GD Steele, Jr., and LB Chen. (1991). Intracellular heterogeneity in mitochondrial membrane potentials revealed by a J-aggregate-forming lipophilic cation JC-1. Proc Natl Acad Sci USA 88:3671-3675.

34. Mamaeva SE. (1998). Karyotyptic evolution of cells in culture: a new concept. Int Rev Cytol 178:1-40.

35. Hiyama E and Hiyama K. (2007). Telomere and telomerase in stem cells. Br J Cancer 96:1020-1024.

36. Collins K and JR Mitchell. (2002). Telomerase in the human organism. Oncogene 21:564-579.

37. Blasco MA. (2005). Telomeres and human disease: ageing, cancer and beyond. Nat Rev Genet 6:611-622.

38. Wright WE and JW Shay. (2005). Telomere biology in aging and cancer. J Am Geriatr Soc 53(9 Suppl):S292-S294.

39. Park YB, YY Kim, SK Oh, SG Chung, SY Ku, SH Kim, YM Choi and SY Moon. (2008). Alterations of proliferative and differentiation potentials of human embryonic stem cells during long-term culture. Exp Mol Med 40:98108.

40. Park S, KS Lee, YJ Lee, HA Shin, HY Cho, KC Wang, YS Kim, HT Lee, KS Chung, EY Kim and J Lim. (2004). Generation of dopaminergic neurons in vitro from human embryonic stem cells treated with neurotrophic factors. Neurosci Lett 359:99-103.

41. Harman D. (1956). Aging: a theory based on free radical and radiation chemistry. J Gerontol 11:298-300.

42. Trifunovic A, A Wredenberg, M Falkenberg, JN Spelbrink, AT Rovio, CE Bruder, YM Bohlooly, S Gidlof, A Oldfors, R Wibom, J Tornell, HT Jacobs and NG Larsson. (2004). Premature ageing in mice expressing defective mitochondrial DNA polymerase. Nature 429:417-423. 
43. Linnane AW, S Marzuki, T Ozawa, et al. (1989). Mitochondrial DNA mutations as an important contributor to ageing and degenerative diseases. Lancet 1:642-645.

44. Brenner CA, HM Kubisch and KE Pierce. (2004). Role of the mitochondrial genome in assisted reproductive technologies and embryonic stem cell-based therapeutic cloning. Reprod Fertil Dev 16:743-751.

45. Schieke SM, M Ma, L Cao, McCoy JP, C Liu, NF Hensel, AJ Barrett, M Boehm and T Finkel. (2008). Mitochondrial metabolism modulates differentiation and teratoma formation capacity in mouse embryonic stem cells. J Biol Chem 283: 28506-28512.

46. Nesti C, L Pasquali, F Vaglini, G Siciliano and L Murri. (2007). The role of mitochondria in stem cell biology. Bioscience reports. 27:165-171.

47. St. John JC, Ramalho-Santos J, HL Gray, P Petrosko, VY Rawe, CS Navara, CR Simerly and GP Schatten. (2005). The expression of mitochondrial DNA transcription factors during early cardiomyocyte in vitro differentiation from human embryonic stem cells. Cloning Stem Cells 7:141-153.

48. St. John J and R Lovell-Badge. (2007). Human-animal cytoplasmic hybrid embryos, mitochondria, and an energetic debate. Nat Cell Biol 9:988-992.

49. Rivolta MN and MC Holley. (2002). Asymmetric segregation of mitochondria and mortalin correlates with the multi-lineage potential of inner ear sensory cell progenitors in vitro. Brain Res Dev Brain Res 133:49-56.
50. Ezashi T, P Das and RM Roberts. (2005). Low O2 tensions and the prevention of differentiation of hES cells. Proc Natl Acad Sci USA 102:4783-4788.

51. Sergey SK, PS Vladimir and AS Anatoly. (1997). High protonic potential actuates a mechanism of production of reactive oxygen species in mitochondria. FEBS Lett 416:15-18.

Address correspondence to: Dr. Joseph C. Wu Department of Medicine

Stanford University School of Medicine 300 Pasteur Drive, Grant S140 Stanford, CA 94305-5111

E-mail: joewu@stanford.edu

Dr. Xuetao Pei

Stem Cell and Regenerative Medicine Lab Beijing Institute of Transfusion Medicine 27 Taiping Road Beijing 100850

China

E-mail: peixt@nic.bmi.ac.cn

Received for publication November 22, 2009

Accepted after revision July 12, 2010

Prepublished on Liebert Instant Online July 14, 2010 